\title{
COMPARAÇÕES DE MEDIDAS DE QUALIDADE DE VIDA ENTRE MULHERES E HOMENS EM HEMODIÁLISE
}

\author{
Gildete Barreto lopes, Maria tereza Silveira Martins, Cácia Mendes Matos, Josete leão de Amorim, Eneida Barreto leite, Eva Alves \\ Miranda, antonio alberto lopes* \\ Trabalho realizado na Faculdade de Medicina da Universidade Federal da Bahia \\ Coordenação central - Núcleo de Epidemiologia Clínica do Hospital Universitário Professor Edgard Santos - Salvador, BA.
}

\section{"Correspondêncio}

Rua Mar. Floriano 448, apt

1301, Canela - Salvador - BA

Cep 40110-010

aaslopes@ufba.br

\section{RESUMO}

ОвјEтivo. Comparar homens e mulheres, tratados cronicamente por hemodiálise, quanto a escores de qualidade de vida relacionada com saúde (QVRS) e avaliar potenciais influências da idade e comorbidades.

Métodos. Corte transversal dos dados da linha de base de 254 mulheres e 349 homens participantes do estudo PROHEMO, sendo desenvolvido em unidades de diálise de Salvador. Utilizando a versão do Kidney Disease Quality of Life Short Form (KDQOL-SF), validada para a população brasileira, foram determinados os escores das escalas: sumário do componente físico [PCS], sumário do componente mental [MCS] e sintomas/problemas relacionados à doença renal. Os escores do PCS e MCS foram determinados utilizando as oito escalas genéricas do KDQOL-SF. Regressão linear múltipla foi usada para estimar diferenças de escores (DE) entre homens e mulheres, ajustadas para idade, anos em hemodiálise, insuficiência cardíaca, doença cerebrovascular, diabetes, doença arterial periférica e níveis séricos de hemoglobina e albumina.

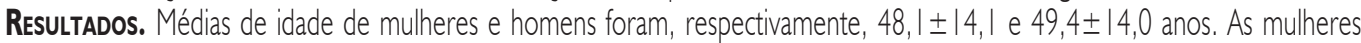
apresentaram menores escores em todas as escalas genéricas de QVRS. Apresentaram também médias significantemente menores $(\mathrm{P}<0.01$ ) no PCS ( $\mathrm{DE}=2,4$ pontos), $\mathrm{MCS}$ (DE $=3$ pontos) e sintomas/problemas ( $\mathrm{DE}=6,6$ pontos). As diferenças foram independentes dos efeitos de comorbidades e mais amplas no grupo com idade superior a 49 anos.

Conclusäo. Os dados indicam que em várias escalas de QVRS as mulheres tratadas cronicamente por hemodiálise apresentam menores escores do que os homens. Os menores escores de QVRS em mulheres foram observados em diferentes grupos etários, independente de comorbidades.

Unitermos: Qualidade de vida. Sexo. Gênero e saúde. Grupos etários. Hemodiálise. Doença renal crônica.

\section{INTRODUÇÃO}

Reconhecendo a necessidade de melhorar a qualidade de vida relacionada com a saúde (QVRS) dos pacientes tratados cronicamente por hemodiálise, o Instituto de Medicina dos Estados Unidos e a National Kidney Foundation, por meio do Kidney Disease Outcome Quality Initiative (K/DOQI), recomendam avaliações sistemáticas de escores de QVRS destes pacientes como um dos parâmetros de adequação do tratamento'. Além de informar sobre o impacto do tratamento no bem-estar dos pacientes, a avaliação da QVRS ajuda a identificar aqueles com risco maior de eventos adversos ${ }^{2-5}$.

Os resultados de diversos estudos sugerem que, no curso de determinadas doenças, as mulheres são mais afetadas do que os homens na QVRS 6-10. Os dados do DOPPS, um estudo em pacientes tratados cronicamente por hemodiálise em diversos países, também indicam que as mulheres apresentam menores níveis no aspecto físico de QVRS e maior incômodo devido a sintomas de insuficiência renal do que os homens, independente do efeito de um grande número de co-variáveis ${ }^{5}$. No Brasil, existe carência de estudos especialmente delineados para comparar escores de QVRS entre homens e mulheres tratados cronicamente por hemodiálise, levando em consideração os potenciais efeitos de comorbidades e outras importantes co-variáveis, como idade e concentrações séricas de hemoglobina e albumina.

O presente estudo utiliza dados da linha de base de uma coorte prospectiva de pacientes em hemodiálise crônica, que vem sendo desenvolvida em clinicas de diálise da cidade de Salvador, com o objetivo de comparar homens e mulheres de diferentes grupos etários quanto a escores de QVRS, no que se refere ao aspecto físico, ao aspecto mental e ao incômodo devido a sintomas/ problemas relacionados com a uremia e o tratamento por hemodiálise crônica. Também avaliamos se a idade, a prevalência de comorbidades e as concentrações plasmáticas de albumina e hemoglobina interferem nas comparações de escores de QVRS entre homens e mulheres.

\section{Métodos}

Corte transversal de dados da linha de base, i.e., no início da coleta dos dados do "Estudo prospectivo do prognóstico de pacientes tratados cronicamente por hemodiálise" (Estudo PROHEMO), desenvolvido em Salvador. Os dados analisados são de quatro clínicas participantes do estudo. O PROHEMO foi aprovado pelo Comitê de Ética em Pesquisa da Faculdade de Medicina da Universidade Federal da Bahia. Todos os pacientes assinaram o termo de consentimento livre e esclarecido para participação no estudo. 


\begin{tabular}{|c|c|c|c|}
\hline Característica & Mulheres & Homens & Valor de P \\
\hline Idade (média $\pm D P)$ & $48,| \pm| 4, \mid$ & $49,4 \pm 14,0$ & 0,252 \\
\hline Albumina sérica(média ニDP) & $3,9 \pm 0,5$ & $4,0 \pm 0,5$ & 0,028 \\
\hline Hemoglobina sérica(média $\pm D P$ ) & $10,1 \pm 1,7$ & $10,4 \pm 1,7$ & 0,021 \\
\hline \% Insuficiência cardíaca & 10,9 & 13,5 & 0,351 \\
\hline$\%$ Diabetes & 20,5 & 14,9 & 0,077 \\
\hline \% Doença cerebrovascular & 5,3 & 4,5 & 0,662 \\
\hline \% Doença vascular periférica & 4,9 & 4,2 & 0,695 \\
\hline Anos em diálise (mediana) & 3,9 & 3,8 & 0,827 \\
\hline
\end{tabular}

Foram analisados dados de 603 pacientes adultos, 254 mulheres e 349 homens. Para avaliar QVRS utilizou-se a versão do Kidney Disease Quality of Life Short Form (KDQOL-SF), traduzida para a língua portuguesa e validada para a população brasileira"1, 12. O KDQOL-SF combina escalas específicas para pacientes com doença renal crônica e pacientes tratados cronicamente por diálise com as oito escalas genéricas originárias do SF-36, ou seja, capacidade física, limitação de atividade por problema físico, dor, saúde geral, bem-estar emocional (saúde mental), limitação por problemas emocionais, funcionamento social e vitalidade (energia/fadiga) ${ }^{13}$. Maiores escores nas escalas do KDQOL são interpretados como melhor nível de QVRS. Utilizando um algoritmo proposto por Ware et al., que toma por base as oito escalas originais do SF-36, foram determinados escores do sumário do componente físico (Physical component summary ou PCS) e o sumário do componente mental (Mental component summary ou MCS) $)^{14}$. Para avaliar a escala sintomas/problemas do componente renal do KDQOL-SF, os pacientes foram questionados quanto ao nível de incômodo, nas últimas quatro semanas, referente a: dores musculares, dor no peito, câimbras, prurido, pele seca, dispnéia, fraqueza/tontura, anorexia, esgotamento/cansaço, dormência nas mãos ou pés, náuseas, mialgia e problemas com acesso vascular. Para determinar o escore de sintomas/problemas, foi utilizado o algoritmo proposto por Hays et al. ${ }^{13}$. A presença de comorbidades foi anotada pelo nefrologista em questionário desenvolvido para o PROHEMO. Os dados laboratoriais foram os mais próximos da data de entrada do paciente no estudo.

Modelos de regressão linear múltipla foram utilizados para estimar diferenças entre homens e mulheres nos escores (DE) de cada medida de QVRS, ajustados para efeito de confusão de diversas co-variáveis. As seguintes co-variáveis foram incluídas no modelos de regressão linear: idade, número de anos em diálise, hemoglobina, albumina, diabetes, insuficiência cardíaca, doença cérebro vascular e doença vascular periférica. Foram também utilizados modelos de regressão linear múltipla com coeficiente de interação do sexo com a idade, para avaliar se as comparações dos escores de QVRS das mulheres com os homens variavam significantemente entre os grupos etários $<50$ anos e ${ }^{3} 50$ anos.

\section{Resultados}

A Tabela I mostra as características dos homens e das mulhe-

\section{Tabela 2 - Comparaçóes dos escores de escalas de qualidade de vida relacionada com a saúde de mulheres e homens na amostra total e por faixas etárias}

\begin{tabular}{|c|c|c|c|c|}
\hline \multicolumn{3}{|c|}{$\begin{array}{l}\text { Escalas de qualidade de vida } \\
\text { relacionadas com saúde } \\
\end{array}$} & \multicolumn{2}{|c|}{$\begin{array}{l}\text { Diferença de escores entre } \\
\text { homens e mulheres }\end{array}$} \\
\hline & Mulheres & Homens & Não ajustada & Ajustada \\
\hline \multicolumn{5}{|c|}{ Grupo total, N=603 } \\
\hline $\begin{array}{l}\text { Sumário do componente } \\
\text { físico (PCS) }\end{array}$ & $38,4 \pm 10,2$ & $40,8 \pm 10,2$ & $\begin{array}{c}2,4 \\
P=0,005\end{array}$ & $\begin{array}{c}2,3 \\
P=0,00\end{array}$ \\
\hline $\begin{array}{l}\text { Sumário do componente } \\
\text { mental(MCS) }\end{array}$ & $46,0 \pm 13,3$ & $49,0 \pm 11,6$ & $\begin{array}{c}3 \\
P=0,005\end{array}$ & $\begin{array}{c}32,9 \\
P=0,005\end{array}$ \\
\hline Sintomas/Problemas & $72,6 \pm 18,3$ & $79,2 \pm 18,3$ & $\begin{array}{c}6,6 \\
P<0,001\end{array}$ & $\begin{array}{c}6,3 \\
P<0,001\end{array}$ \\
\hline
\end{tabular}

Idade $<50$ anos, $\mathrm{N}=325$

$\begin{array}{ccccc}\text { Sumário do componente } & 40,3 \pm 9,4 & 42,6 \pm 10,0 & \mathbf{2 , 3} & \mathbf{2 , 2} \\ \text { Físico(PCS) } & & & P=0,033 & P=0,046 \\ \text { Sumário do componente } & 45,8 \pm 12,5 & 48,3 \pm 11,6 & \mathbf{2 , 5} & \mathbf{2 , 3} \\ \text { mental(MCS) } & & P=0,066 & P=0,088 \\ \text { Sintomas/Problemas } & 74,7 \pm 17,8 & 80,4 \pm 17,2 & \mathbf{5 , 7} & \mathbf{5 , I} \\ & & & P<0,002 & P<0,011\end{array}$

Idade $\geq \mathbf{5 0}$ anos, $\mathrm{N}=\mathbf{2 7 8}$

$\begin{array}{ccccc}\text { Sumário do componente } & 35,8 \pm 10,7 & 38,8 \pm 10,1 & 3,0 & \mathbf{2 , 6} \\ \text { físico(PCS) } & & P=0,021 & P=0,046 \\ \text { Sumário do componente } & 46,4 \pm \mid 4,3 & 49,8 \pm 11,7 & \mathbf{3 , 4} & \mathbf{3 , 5} \\ \text { mental(MCS) } & & P=0,03 \mid & P=0,026 \\ \text { Sintomas/Problemas } & 69,8 \pm 18,5 & 77,9 \pm 16,5 & \mathbf{8 , 3} & \mathbf{7 , 7} \\ & & P<0,00 \mid & P<0,001\end{array}$

* As comparações foram ajustadas para as co-variáveis listadas na Tabela I, utillizando

regressão linearmútipla

res. As médias das concentrações séricas de albumina e hemoglobina foram um pouco menores em mulheres $(P<0,05)$. Observou-se também, maior prevalência de diabetes melito em mulheres, porém a diferença foi marginalmente significante.

$\mathrm{Na}$ amostra total, os escores de PCS variaram entre 14,6 e 60,7 (média $=39,7 \pm 10,3 ;$ mediana $=40,8$ ), MCS entre 14,2 e 75,0 (média $=47,7 \pm 12,4$; mediana $=48,6$ ) e sintomas $/$ problemas entre 12,5 e 100 (média=76,4 1 17,8; mediana=81,2). Conforme a Tabela 2, os escores de PCS, MCS e sintomas/ problemas foram significantemente menores em mulheres do que em homens. $\mathrm{Na}$ análise não ajustada para co-variáveis, as diferenças entre mulheres e homens foram de 2,4 pontos para $\operatorname{PCS}(P=0,005)$, três pontos para $\operatorname{MCS}(P=0,005)$ e 6,6 pontos para sintomas/problemas $(\mathrm{P}<0,00 \mathrm{I})$. $\mathrm{Na}$ análise de fatores potencialmente confundidores da associação entre sexo e medidas de QVRS, observou-se que idade mais avançada, níveis mais baixos de hemoglobina e DVP foram significantemente associados a escores mais baixos de PCS e sintomas/problemas. Diabetes melito e DCV foram também significantemente associados a escores mais baixos de PCS. Insuficiência cardíaca foi significantemente associada a escores mais baixos de MCS e de sintomas/problemas. Após 
LOPES GB ET AL.

ajustes para estas características, as diferenças entre homens e mulheres permaneceram estatisticamente significantes e similares às observadas na análise não ajustada (Tabela 2). Procuramos identificar quais as escalas do SF-36 poderiam contribuir para os escores mais baixos do PCS e MCS em mulheres. Observamos que as médias dos escores foram menores em mulheres nas oito escalas originais do SF-36 (as diferenças entre mulheres e homens variaram de 3,3 pontos para saúde geral a 10,4 pontos para limitação por problemas emocionais), sendo estatisticamente significantes para capacidade física, dor, bem-estar emocional (saúde mental), limitação por problemas emocionais e vitalidade (energia/ fadiga).

A Tabela 2 mostra as comparações de escores de QVRS entre mulheres e homens em dois grupos etários: $<50$ e ${ }^{3} 50$ anos. As diferenças de escores entre mulheres e homens foram mais amplas no grupo ${ }^{3} 50$ anos, no entanto os coeficientes de interação do sexo com a idade nos modelos de regressão linear múltipla não foram estatisticamente significantes (valores de P superiores a 0,4).

\section{Discussão}

Os resultados do presente estudo mostram que as mulheres tratadas por hemodiálise crônica na cidade de Salvador apresentam níveis mais baixos de QVRS, tanto no aspecto físico quanto no mental. Os dados também indicam que o incômodo devido a sintomas e problemas relacionados com a uremia e o tratamento por hemodiálise crônica é maior em mulheres do que em homens. Estes achados que observamos na cidade de Salvador são consistentes com os resultados do DOPPS em pacientes tratados por hemodiálise nos Estados Unidos, países da Europa e Japãa ${ }^{5}$. No DOPPS, as mulheres também apresentaram escores mais baixos de PCS, MCS e sintomas/problemas. No entanto, as diferenças observadas em nosso estudo foram bem mais amplas do que no DOPPS.

No presente estudo, idade mais avançada, níveis mais baixos de hemoglobina, presença de insuficiência cardíaca, diabetes, doença cerebrovascular e doença vascular periférica estiveram associados a escores mais baixos de pelo menos uma das medidas de QVRS que avaliamos. Apesar dos ajustes para estas variáveis, as diferenças entre os sexos em PCS, MCS e sintomas/problemas praticamente não foram modificadas e permaneceram estatisticamente significantes. Estes achados sugerem que fatores não biológicos podem estar contribuindo para explicar as diferenças de QVRS entre os sexos. Experiências prévias, frustrações devido à expectativas não atendidas e à falta de suporte social são fatores que devem ser considerados como potenciais determinantes das diferenças de QVRS observadas entre mulheres e homens ${ }^{15}$. Além do que, as mulheres são, aparentemente, mais facilmente afetadas psicologicamente pelos estressores ambientais do que os homens $^{16}$. É possível que as mulheres tratadas por hemodiálise crônica, por terem que manter as funções tradicionais, ou seja, a responsabilidade de cuidar da casa e dos filhos, sejam expostas a maior carga de estresse físico e mental.

O método utilizado para determinar os escores do PCS e MCS leva em consideração todas as oito escalas genéricas do KDQOLSF que são originárias do SF-36. É importante notar que as mulheres apresentaram escores mais baixos em todas as escalas genéricas. Estes nossos achados são também consistentes com o que tem sido observado em pacientes tratados por hemodiálise fora do Brasil ${ }^{5,17,18}$. Diferindo destes achados, em estudo desenvolvido em uma única clínica do interior do Ceará, com amostra de 107 pacientes, não se observou diferenças significantes entre os sexos $^{19}$. O menor poder estatístico do estudo do Ceará é uma possível explicação para a ausência de significância estatística nas comparações entre homens e mulheres, um achado que contrasta com as amplas diferenças estatisticamente significantes entre os sexos nos escores das escalas genéricas de QVRS observadas no nosso estudo.

Tem sido sugerido que, com o avançar da idade, os escores de QVRS reduzam de forma mais acentuada em mulheres, presumivelmente devido às alterações hormonais e à depressãa $0^{20}$, ${ }^{21}$. Por esta razão, também comparamos os escores de QVRS de homens e mulheres em dois grupos etários: 18 a 49 e 350 anos. De acordo com os dados, os menores escores de QVRS são observados nas duas faixas etárias, com tendência para aumento das diferenças na faixa etária ${ }^{3} 50$ anos.

Embora o presente estudo ofereça informações importantes para apoiar o desenvolvimento de estratégias visando melhorar a QVRS dos pacientes em hemodiálise, limitações metodológicas inerentes a um estudo de corte transversal devem ser consideradas. A avaliação transversal dos dados impossibilita avaliar se 0 aparecimento de determinada comorbidade antecedeu modificações nos escores de QVRS. Além do mais, o diagnóstico de certas comorbidades, como a insuficiência cardíaca, nem sempre é fácil de ser comprovado em pacientes tratados por hemodiálise.

\section{ConClusão}

O presente estudo chama a atenção para níveis mais baixos de QVRS em mulheres do que em homens, independente da idade e da presença de comorbidades. As diferenças observadas são mais amplas do que o tem sido observado em pacientes tratados em outras regiões. Embora o sexo não seja uma característica modificável, as diferenças observadas entre homens e mulheres tratados cronicamente por hemodiálise são importantes no sentido de identificar pacientes que necessitam de cuidados específicos para melhorar a QVRS.

\section{Agradecimentos}

Os autores agradecem a colaboração das equipes dos centros de diálise participantes do PROHEMO. Este trabalho recebeu apoio do Conselho Nacional de Ciência e Tecnologia, CNPq, processos 484743/2006-6 e 308068/ 2006-8.

Conflito de interesse: não há. 


\section{SUMMARY}

\section{COMPARISONS OF QUALITY OF LIFE MEASURES BETWEEN WOMEN AND MEN ON HEMODIALYSIS}

OBJECTIVE. TO compare men and women on chronic hemodialysis in relation to their scores in health-related quality of life $(H R Q O L)$ and evaluate the potential influence of age and comorbidities on the comparison.

Methods. Cross-section of the baseline data of 254 women and 349 men, participants of the PROHEMO Study that has been developed in dialysis units of Salvador. By using the version of the Kidney Disease Quality of Life Short Form KDQOL-SF, validated for the Brazilian population, the following scores were determined: physical component summary (PCS), mental component summary [MCS] and the scale for symptoms/problems related to renal disease. The PCS and MCS scores were derived from the eight generic KDQOL-SF scales. Multiple linear regression was used to estimate differences in the scores of men and women (DS), adjusted for age, years on hemodialysis, heart failure, cerebrovascular disease, peripheral vasculopathy, diabetes, serum hemoglobin and serum albumin.

RESULTS. Mean ages were $48.1 \pm 14.1$ and $49.4 \pm 14.0$ years for women and men, respectively. Women had lower scores for all generic $H R Q O L$ scales. They also had significantly $(P<0.001)$ lower scores for PCS (DS $=2.4$ points), MCS (DS $=3$ points) and symptoms/problems (DS $=6.6$ points). Differences were independent of comorbidties and greater in the group over 49 years of age.

CONCLUSION. Data indicate that for several scales of HRQOL, women treated chronically by hemodialysis had lower scores than men. Lower scores in women were observed for different age groups, independently of comorbidities. [Rev Assoc Med Bras 2007; 53(6): 506-9]

KEY WORDS: Quality of fife. Gender and health. Age groups. Hemodialysis. Chronic kidney disease.

\section{REFERÊNCIAS}

I. K/DOQI clinical practice guidelines for chronic kidney disease: evaluation, classification, and stratification - Part 6: association of level of GFR with complications in adults Am J Kidney Dis. 2002;39:SI I I-69.

2. Lopes AA, Bragg-Gresham JL, Satayathum S, McCullough K, Pifer T, Goodkin DA, et al. Health-related quality of life and associated outcomes among hemodialysis patients of different ethnicities in the United States: the Dialysis Outcomes and Practice Patterns Study (DOPPS). Am J Kidney Dis. 2003;41:605-I5.

3. Mapes DL, Bragg-Gresham JL, Bommer J, Fukuhara S, McKevitt P, Wikstrom B, et al. Health-related quality of life in the Dialysis Outcomes and Practice Patterns Study (DOPPS). Am J Kidney Dis. 2004;44:5460.

4. Mapes DL, Lopes AA, Satayathum S, McCullough KP, Goodkin DA, Locatelli $F$, et al. Health-related quality of life as a predictor of mortality and hospitalization: the Dialysis Outcomes and Practice Patterns Study (DOPPS). Kidney Int. 2003;64:339-49.
5. Lopes AA, Bragg-Gresham JL, Goodkin DA, Fukuhara S, Mapes DL, Young EW, et al. Factors associated with health-related quality of life among hemodialysis patients in the DOPPS. Qual Life Res. 2007; | 6:545-57.

6. Norris CM, Ghali WA, Galbraith PD, Graham MM, Jensen LA, Knudtson ML. Women with coronary artery disease report worse health-related quality of life outcomes compared to men. Health Qual Life Outcomes. 2004:2:21.

7. Cowling T, Jennings LW, Goldstein RM, Sanchez EQ, Chinnakotla S, Klintmalm GB, et al. Liver transplantation and health-related quality of life: scoring differences between men and women. Liver Transpl. 2004; 10:88-96.

8. Lindquist R, Dupuis G, Terrin ML, Hoogwerf B, Czajkowski S, Herd JA, et al. Comparison of health-related quality-of-life outcomes of men and women after coronary artery bypass surgery through I year: findings from the POST CABG Biobehavioral Study. Am Heart J. $2003 ;$ | 46: 1038-44

9. Hallert C, Sandlund O, Broqvist M. Perceptions of health-related quality of life of men and women living with coeliac disease. Scand J Caring Sci. 2003; 17:301-7.

I 0. Herlitz J, Wiklund I, Sjoland H, Karlson BW, Karlsson T, Haglid M, et al. Relief of symptoms and improvement of health-related quality of life five years after coronary artery bypass graft in women and men. Clin Cardiol. 2001;24:385-92.

I I. Duarte PS, Ciconelli RM, Sesso R. Cultural adaptation and validation of the "Kidney Disease and Quality of Life-Short Form (KDQOL-SF I.3)" in Brazil. Braz J Med Biol Res. 2005;38:261-70.

12. Duarte PS, Miyazaki MC, Ciconelli RM, Sesso R. Tradução e adaptação cultural do instrumento de avaliação de qualidade de vida para pacientes renais crônicos (KDQOL-SF). Rev Assoc Med Bras. 2003;49:375-8I.

13. Hays RD, Kallich JD, Mapes DL, Coons SJ, Carter WB. Development of the kidney disease quality of life (KDQOL) instrument. Qual Life Res. 1994:3:329-38.

14. Ware JE, Kosinski M, Keller SD. SF-36 Physical and mental health summary scales: a user's manual. Boston: New England Medical Center- The Health Institute; 1994.

15. Lunsky Y. Depressive symptoms in intellectual disability: does gender play a role? J Intellect Disabil Res. 2003;47:4I7-27.

16. Hankin BL, Abramson LY. Development of gender differences in depression: an elaborated cognitive vulnerability-transactional stress theory. Psychol Bull. 2001;127:773-96.

17. Mingardi G, Cornalba L, Cortinovis E, Ruggiata R, Mosconi P, Apolone $G$. Health-related quality of life in dialysis patients. A report from an Italian study using the SF-36 Health Survey. DIA-QOL Group. Nephrol Dial Transplant. 1999;14:1503-10.

18. Valderrabano F, Jofre R, Lopez-Gomez JM. Quality of life in end-stage renal disease patients. Am J Kidney Dis. 2001;38:443-64.

19. Santos PR. Relação do sexo e da idade com nível de qualidade de vida em renais crônicos hemodialisados. Rev Assoc Med Bras. 2006; $52: 356-9$.

20. Varvaro FF, Olds NB, Zullo TG, Murali S. Determining Quality of Life in Older and Younger Women With Congestive Heart Failure and Myocardial Infarction. Am J Geriatr Cardiol. 1999;8:15-20.

21. Wojnar M, Drod W, Araszkiewicz A, Szymanski W, NawackaPawlaczyk D, Urbanski R, et al. Assessment and prevalence of depression in women 45-55 years of age visiting gynecological clinics in Poland: screening for depression among midlife gynecologic patients. Arch Womens Ment Health. 2003;6:193-201.

Artigo recebido: 05/04/07

Aceito para publicação: 02/10/07 